\title{
Accounting Program Assessment: Exit Interviews of Graduating Seniors
}

\author{
Barbara R. Stewart, Towson University, USA \\ Charles L. Martin Jr., Towson University, USA \\ Lamont F. Steedle, Towson University, USA
}

\begin{abstract}
Exit interviews of university graduating seniors have been used to a very limited extent when evaluating academic programs. It is our belief that developing and implementing an exit interview plan stimulates faculty dialog and improves ongoing feedback for department program assessment. From 2001 to 2007, the Accounting Department at Towson University has conducted exit interviews with all graduating seniors as part of the annual internal department assessment activities. The exit interviews have served to assist the Department of Accounting in evaluating its performance as judged by the students served. The positive comments assist to reinforce the quality activities of the department, while the negative comments permit the department to become aware of areas of perceived weakness that are in need of improvement. By receiving annual feedback from a valuable, relevant source, the department is able to continuously monitor, adjust, and improve its performance.
\end{abstract}

Keywords: Exit Interviews; Assessment; Graduating Accounting Seniors; Continuous Improvement

\section{INTRODUCTION}

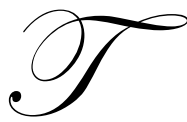

he exit interview has been used for over fifty years in industry (Doll and Jacobs, 1988). It has also found its way into academe when employees are leaving the university. However, exit interviews of university graduating seniors have been used to a very limited extent when evaluating academic programs (Louise, Mares and Calkins, 1986). It is our belief that developing and implementing an exit interview plan stimulates faculty dialog and improves ongoing feedback for department program assessment.

The procedures described here were applied in the Accounting Department at Towson University. Towson is the second largest public university in Maryland with a growing enrollment currently approximating 20,000 students and over 500 faculty members. Its Carnegie classification is that of a Comprehensive University. The Accounting Department faculty is comprised of fourteen full-time members (eleven accounting and three law professionals) and a varying number of adjuncts. The College of Business and Economics is AACSB accredited, and the Accounting Department is also an AACSB Accounting accredited undergraduate program.

For the past seven years, the Accounting Department at Towson University has conducted exit interviews with all graduating seniors as part of the annual internal department assessment activities. The exit interview is conducted by the Accounting Chair as part of the student's graduation clearance. Typically an hour is allocated for the interview, but it may last anywhere from 15 minutes to 2 hours. The interview is held in the Chair's office. It is informal. All questions are open-ended and asked in a dialog format. The exit interview focuses upon three main areas: education, employment, and perceptions of the academic experience. Students are assured of confidentiality.

During the seven-year period from 1999-2000 to 2005-2006, 427 graduating seniors took part in the exit interviews. The number of annual graduates ranged from 49 to 74 , an average of 61 graduates per year. Academic years 2004-2005 and 2005-2006 witnessed a sharp increase in graduates with 74 and 71, respectively.

All interviews were conducted on an individual basis by the Accounting Chair. The same individual held the Accounting Chair position over this time frame, except for one semester (spring 2005), when the Acting Chair conducted the interviews utilizing the same outline of open-ended questions. 
The exit interviews provided the department with invaluable information ranging from the timing of the student's decision to become an accounting major, through the evaluation of the educational experience, to their plans after graduation. The information gained through these interviews has served and will continue to serve the accounting department in fulfilling its mission of preparing students for a successful career in accounting. Furthermore, the information is used as part of the basis for short-term as well as long-term development plans.

Table I: Decision To Major In Accounting

\begin{tabular}{|c|c|c|c|c|c|c|c|c|c|c|}
\hline \multirow[t]{2}{*}{ Academic Year } & \multirow[b]{2}{*}{ Wt Avg } & \multirow[b]{2}{*}{ total \# } & \multicolumn{2}{|c|}{$2005-2006$} & \multicolumn{2}{|c|}{$2004-2005$} & \multicolumn{2}{|c|}{$2003-2004$} & \multicolumn{2}{|c|}{ 2002-2003 } \\
\hline & & & $\#$ & $\%$ & \# & $\%$ & \# & $\%$ & \# & $\%$ \\
\hline \multicolumn{11}{|l|}{ "When Accounting Major Was Decided } \\
\hline High School & $33 \%$ & 83 & 27 & $38 \%$ & 19 & $26 \%$ & 17 & $35 \%$ & 20 & $34 \%$ \\
\hline Freshman Year & $21 \%$ & 53 & 11 & $15 \%$ & 15 & $20 \%$ & 14 & $29 \%$ & 13 & $22 \%$ \\
\hline Sophomore Year & $27 \%$ & 67 & 19 & $27 \%$ & 24 & $32 \%$ & 7 & $14 \%$ & 17 & $29 \%$ \\
\hline Junior Year & $12 \%$ & 30 & 8 & $11 \%$ & 7 & $9 \%$ & 8 & $16 \%$ & 7 & $12 \%$ \\
\hline Senior Year & $1 \%$ & 3 & 1 & $1 \%$ & 1 & $1 \%$ & & & 1 & $2 \%$ \\
\hline \multirow{2}{*}{ After First Degree } & $6 \%$ & 16 & $\underline{5}$ & $7 \%$ & $\underline{8}$ & $11 \%$ & $\underline{3}$ & $6 \%$ & & \\
\hline & $100 \%$ & $\underline{252}$ & $\underline{71}$ & $100 \%$ & $\underline{74}$ & $100 \%$ & $\underline{49}$ & $100 \%$ & 58 & $100 \%$ \\
\hline \multicolumn{11}{|l|}{ Previous Major } \\
\hline Business Administration & $27 \%$ & 46 & 13 & $30 \%$ & 11 & $25 \%$ & 8 & $33 \%$ & 14 & $37 \%$ \\
\hline Computer Science/Engineering/Math & $16 \%$ & 27 & 8 & $18 \%$ & 13 & $30 \%$ & 3 & $13 \%$ & 3 & $8 \%$ \\
\hline Finance & $5 \%$ & 9 & 1 & $2 \%$ & 8 & $18 \%$ & & & & \\
\hline Economics & $4 \%$ & 6 & 1 & $2 \%$ & 3 & $7 \%$ & 1 & $4 \%$ & 1 & $3 \%$ \\
\hline Education & $4 \%$ & 6 & 1 & $2 \%$ & 2 & $5 \%$ & 3 & $13 \%$ & & \\
\hline Mass Communications & $4 \%$ & 6 & 2 & $5 \%$ & 1 & $2 \%$ & 2 & $8 \%$ & 1 & $3 \%$ \\
\hline Nursing/Physical Therapy/Exercise Science & $4 \%$ & 6 & 1 & $2 \%$ & 2 & $5 \%$ & 2 & $8 \%$ & 1 & $3 \%$ \\
\hline Biology/Pre-Med & $3 \%$ & 5 & 2 & $5 \%$ & 1 & $2 \%$ & & & 2 & $5 \%$ \\
\hline English & $2 \%$ & 4 & 2 & $5 \%$ & & & & & 2 & $5 \%$ \\
\hline Psychology & $2 \%$ & 3 & 1 & $2 \%$ & & & 1 & $4 \%$ & 1 & $3 \%$ \\
\hline Horticulture & $1 \%$ & 2 & & & 1 & $2 \%$ & & & 1 & $3 \%$ \\
\hline International Studies & $1 \%$ & 2 & 1 & $2 \%$ & & & & & 1 & $3 \%$ \\
\hline Environmental Science & $1 \%$ & 1 & & & 1 & $2 \%$ & & & & \\
\hline General Studies & $1 \%$ & 1 & 1 & $2 \%$ & & & & & & \\
\hline Graphic Design & $1 \%$ & 1 & & & & & & & 1 & $3 \%$ \\
\hline Hotel Administration & $1 \%$ & 1 & & & & & 1 & $4 \%$ & & \\
\hline Pre-Law & $1 \%$ & 1 & & & & & 1 & $4 \%$ & & \\
\hline \multirow[t]{2}{*}{ Undecided } & $25 \%$ & $\underline{42}$ & $\underline{10}$ & $23 \%$ & $\underline{12}$ & $27 \%$ & $\underline{10}$ & $42 \%$ & $\underline{10}$ & $\underline{26 \%}$ \\
\hline & $100 \%$ & $\overline{169}$ & 44 & $100 \%$ & $\overline{55}$ & $125 \%$ & 32 & $\overline{133 \%}$ & $\overline{38}$ & $100 \%$ \\
\hline \multirow[t]{2}{*}{ Decided in High School } & & $\mathbf{8 3}$ & 27 & & 19 & & 17 & & $\underline{20}$ & \\
\hline & & $\overline{252}$ & $\overline{71}$ & & $\overline{74}$ & & $\overline{49}$ & & $\overline{58}$ & \\
\hline \multicolumn{11}{|l|}{ Reason to Change to Accounting Major } \\
\hline Accounting Principles Class & $37 \%$ & 63 & 14 & $32 \%$ & 21 & $38 \%$ & 10 & $32 \%$ & 18 & $47 \%$ \\
\hline Career Opportunities & $37 \%$ & 63 & 17 & $39 \%$ & 23 & $42 \%$ & 15 & $48 \%$ & 8 & $21 \%$ \\
\hline Work Experience & $15 \%$ & 26 & 7 & $16 \%$ & 8 & $15 \%$ & & & 11 & $29 \%$ \\
\hline Friends/Family Advice & $8 \%$ & 13 & 6 & $14 \%$ & 3 & $5 \%$ & 3 & $10 \%$ & 1 & $3 \%$ \\
\hline High school Accounting & $2 \%$ & 3 & & & & & 3 & $10 \%$ & & \\
\hline \multirow[t]{2}{*}{ Upper-level Accounting Class } & $1 \%$ & $\underline{1}$ & & & & & 1 & $\underline{3 \%}$ & & \\
\hline & $100 \%$ & 169 & 44 & $100 \%$ & 55 & $100 \%$ & 32 & $103 \%$ & 38 & $100 \%$ \\
\hline Total Number of Graduates & & 252 & & 71 & & 74 & & 49 & & 58 \\
\hline
\end{tabular}

\section{Decision to Become an Accounting Major (Table I)}

Beginning in 2002-2003 the interviews included questions regarding the choice of the accounting major. Summarized data are shown in Table I. We found that $33 \%$ of the students made their decision to major in accounting while in high school. 
The Maryland Association of Certified Public Accountants (MACPA) makes a concerted effort to talk to students while they are still in high school. The MACPA offers a guest speaker program where members of the Association willingly volunteer to visit local high schools. The Association has enrolled a large student affiliate membership of both high school and college students. The student affiliates receive an online newsletter, Tomorrows CPA, and are invited to many Association functions. This educational program is used to inform students about the accounting profession and specifically the path to obtain CPA licensure. Teachers and guidance/career counselors serve as the primary conduit. The program's information is located at http://www.tomorrowscpa.org/join/htm.

Of those students who had not already committed to accounting in high school, $25 \%$ entered college officially "undecided," $36 \%$ switched to accounting from a previously declared major in the College of Business and Economics (mainly Business

Administration), and $16 \%$ had previously majored in quantitative disciplines such as computer science, engineering or math. The rest came from a wide variety of liberal arts and pre-professional programs.

Through the efforts of the MACPA and Accounting Principles instructors, students have become more aware of the opportunities afforded to accounting graduates. The Accounting Department has targeted strong Accounting Principles students to become accounting majors by inviting these students to attend the annual Accounting Awards Banquet free of charge and by sending high achieving Principles students letters congratulating them on their success and encouraging them to consider a major in accounting. Thirty-seven percent of the students changing to accounting cited the Accounting Principles class as the reason, and another 37\%indicated career opportunities. Together, students' perceived career opportunities and present work experience accounted for 52\% of those deciding to change their major to accounting.

Table II: Accounting Entrance Exam

\begin{tabular}{|c|c|c|c|c|c|c|c|c|c|c|c|c|}
\hline \multirow[t]{2}{*}{ Academic Year } & \multirow[b]{2}{*}{ Wt Avg } & \multirow[b]{2}{*}{ total \# } & \multicolumn{2}{|c|}{ 2003-2004 } & \multicolumn{2}{|c|}{$2002-2003$} & \multicolumn{2}{|c|}{ 2001-2002 } & \multicolumn{2}{|c|}{ 2000-2001 } & \multicolumn{2}{|c|}{$1999-2000$} \\
\hline & & & $\#$ & $\%$ & $\#$ & $\%$ & $\#$ & $\%$ & $\#$ & $\%$ & $\#$ & $\%$ \\
\hline \multicolumn{13}{|l|}{ APAT Test Attempts } \\
\hline One & $75 \%$ & 209 & 36 & $77 \%$ & 41 & $71 \%$ & 42 & $69 \%$ & 45 & $71 \%$ & 45 & $88 \%$ \\
\hline Two & $18 \%$ & 51 & 9 & $19 \%$ & 13 & $22 \%$ & 12 & $20 \%$ & 12 & $19 \%$ & 5 & $10 \%$ \\
\hline Three & $5 \%$ & 15 & 1 & $2 \%$ & 4 & $7 \%$ & 3 & $5 \%$ & 6 & $10 \%$ & 1 & $2 \%$ \\
\hline Four & $1 \%$ & 4 & 1 & $2 \%$ & & & 3 & $5 \%$ & & & & \\
\hline Five & $\underline{0 \%}$ & $\underline{1}$ & & & & & 1 & $2 \%$ & & & & \\
\hline After exam withdrawn & $100 \%$ & 280 & $\begin{array}{c}47 \\
\frac{2}{49}\end{array}$ & $100 \%$ & 58 & $100 \%$ & 61 & $100 \%$ & 63 & $100 \%$ & 51 & $100 \%$ \\
\hline Total Number of Graduates & & 282 & & 49 & & 58 & & 61 & & 63 & & 51 \\
\hline
\end{tabular}

\section{Entrance into the Accounting Major (Table II)}

Beginning in the early 1980s, in addition to specific course requirements, the Department of Accounting required all pre-accounting majors to take and pass $\left(50^{\text {th }}\right.$ percentile) the American Institute of Certified Public Accountant's (AICPA) Level I exam in order to gain entrance into the accounting program.

The Level I test was replaced by the Accounting Program Aptitude Test (APAT), which was developed by and administered under the auspices of the Psychological Corporation. The 90 minute, 75 multiple-choice test covered financial accounting (60\%) and managerial accounting (40\%). The percentile scores of our students on this new test were much higher than those on the Level I, so the minimum-passing percentile was set at 75 . The APAT remained in effect as an entrance requirement into Towson University's accounting program until 2003, when it was withdrawn by the Psychological Corporation. As shown in Table II, of the students who took this test, 75\% passed it on the first attempt. Over $92 \%$ passed the test on the first or second attempt. The department also relied on academic performance for admission into the program. The student had to have a 2.50 or higher overall GPA and a 2.50 or higher GPA in 9 hours or more of accounting courses in order to be admitted into the accounting program. Admission is usually based upon the student's grades in Principles of Accounting I and II and Intermediate I. 
Table III: Credit Hours Completed

Academic Year

\begin{tabular}{|l|l|l|l|l|l|l|}
\hline 2005-2006 & 2004-2005 & 2003-2004 & 2002-2003 & 2001-2002 & 2000-2001 & 1999-2000 \\
\hline
\end{tabular}

\begin{tabular}{|c|c|c|c|c|c|c|c|c|c|c|c|c|c|c|c|}
\hline & Wt Avg & \# & $\%$ & \# & $\%$ & \# & $\%$ & \# & $\%$ & \# & $\%$ & \# & $\%$ & \# & $\%$ \\
\hline \multicolumn{16}{|l|}{ \# hours completed } \\
\hline Over 150 & $38 \%$ & & $31 \%$ & & $36 \%$ & 29 & $59 \%$ & 24 & $41 \%$ & 28 & $46 \%$ & 17 & $27 \%$ & 15 & $29 \%$ \\
\hline 2nd Bach program & & 5 & & 8 & & & & & & & & & & & \\
\hline Changed major & & 11 & & 13 & & & & & & & & & & & \\
\hline FPLN track & & 4 & & & & & & & & & & & & & \\
\hline Minor & & 1 & & 1 & & & & & & & & & & & \\
\hline Double major & & 1 & & 5 & & & & & & & & & & & \\
\hline $140-149$ & $14 \%$ & & $10 \%$ & & $14 \%$ & & $8 \%$ & & $16 \%$ & & $11 \%$ & & $22 \%$ & & $18 \%$ \\
\hline Taking Towson UG & & 1 & & 6 & & 4 & & 2 & & 5 & & 9 & & 5 & \\
\hline Taking UG Other & & 1 & & 2 & & & & 2 & & 1 & & 4 & & 2 & \\
\hline Grad School & & 4 & & 2 & & & & 1 & & & & & & & \\
\hline Going out of State & & & & & & & & 1 & & 1 & & & & 1 & \\
\hline Not Taking CPA & & 1 & & & & & & 2 & & & & 1 & & 1 & \\
\hline \multicolumn{16}{|l|}{ Undecided } \\
\hline $130-139$ & $19 \%$ & & $18 \%$ & & $23 \%$ & & $14 \%$ & & $22 \%$ & & $18 \%$ & & $16 \%$ & & $20 \%$ \\
\hline Taking Towson UG & & 3 & & 3 & & & & 4 & & 4 & & 1 & & 4 & \\
\hline Taking UG Other & & 3 & & 4 & & & & 2 & & & & 1 & & & \\
\hline Grad School & & 4 & & 5 & & 4 & & 4 & & 5 & & 4 & & 2 & \\
\hline Going Out of State & & 2 & & 2 & & 1 & & 1 & & 1 & & 2 & & 2 & \\
\hline Not Taking CPA & & & & 2 & & 2 & & & & 1 & & 2 & & 2 & \\
\hline Undecided & & 1 & & 1 & & & & 2 & & & & & & & \\
\hline $120-129$ & $29 \%$ & & $41 \%$ & & $27 \%$ & & $18 \%$ & & $21 \%$ & & $25 \%$ & & $35 \%$ & & $33 \%$ \\
\hline Taking Towson UG & & 4 & & 1 & & 3 & & 1 & & 4 & & 2 & & 3 & \\
\hline Taking UG Other & & 1 & & 4 & & & & 2 & & 2 & & 2 & & & \\
\hline Grad School & & 16 & & 11 & & 3 & & 4 & & 3 & & 9 & & 6 & \\
\hline Going Out of State & & 4 & & 1 & & & & 1 & & 1 & & 5 & & 1 & \\
\hline Not Taking CPA & & 2 & & 2 & & & & 4 & & 4 & & 4 & & 7 & \\
\hline Undecided & & $\underline{2}$ & & $\underline{1}$ & & $\underline{3}$ & & & & $\underline{1}$ & & & & & \\
\hline & $100 \%$ & $\overline{71}$ & $100 \%$ & 74 & $100 \%$ & $\overline{49}$ & $100 \%$ & 58 & $100 \%$ & $\overline{61}$ & $100 \%$ & 63 & $100 \%$ & 51 & $100 \%$ \\
\hline \multicolumn{16}{|c|}{ 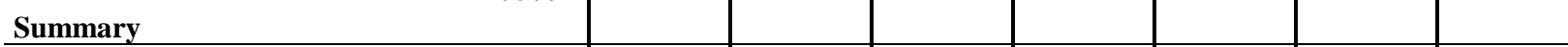 } \\
\hline 150 Hours Met & $38 \%$ & 22 & $31 \%$ & 27 & $36 \%$ & 29 & $59 \%$ & 24 & $41 \%$ & 28 & $46 \%$ & 17 & $27 \%$ & 15 & $29 \%$ \\
\hline Need Additional Courses & $44 \%$ & 37 & $52 \%$ & 38 & $51 \%$ & 14 & $29 \%$ & 22 & $38 \%$ & 24 & $39 \%$ & 32 & $51 \%$ & 22 & $43 \%$ \\
\hline Going Out of State & $6 \%$ & 6 & $8 \%$ & 3 & $4 \%$ & 1 & $2 \%$ & 3 & $5 \%$ & 3 & $5 \%$ & 7 & $11 \%$ & 4 & $8 \%$ \\
\hline Not Taking CPA & $9 \%$ & 3 & $4 \%$ & 4 & $5 \%$ & 2 & $4 \%$ & 6 & $10 \%$ & 5 & $8 \%$ & 7 & $11 \%$ & 10 & $20 \%$ \\
\hline \multirow[t]{2}{*}{ Undecided } & $\underline{3 \%}$ & $\underline{3}$ & $\underline{4 \%}$ & $\underline{2}$ & $\underline{3 \%}$ & $\underline{3}$ & $\underline{6 \%}$ & $\underline{3}$ & $\underline{5 \%}$ & $\underline{1}$ & $\underline{2 \%}$ & $\underline{0}$ & $\underline{0 \%}$ & $\underline{0}$ & $\underline{0 \%}$ \\
\hline & $\overline{100 \%}$ & $\overline{71}$ & $1 \overline{00 \%}$ & $\overline{74}$ & $100 \%$ & $\overline{49}$ & $100 \%$ & $\overline{58}$ & $1 \overline{00 \%}$ & $\overline{61}$ & $100 \%$ & $\overline{63}$ & $1 \overline{00 \%}$ & $\overline{51}$ & $100 \%$ \\
\hline \multicolumn{16}{|l|}{ Need Additional Courses } \\
\hline Taking Towson UG & $37 \%$ & 8 & $22 \%$ & 10 & $26 \%$ & 7 & $50 \%$ & 7 & $32 \%$ & 13 & $54 \%$ & 12 & $38 \%$ & 12 & $55 \%$ \\
\hline Taking UG Other & $17 \%$ & 5 & $14 \%$ & 10 & $26 \%$ & 0 & & 6 & $27 \%$ & 3 & $13 \%$ & 7 & $22 \%$ & 2 & $9 \%$ \\
\hline \multirow[t]{2}{*}{ Grad School } & $46 \%$ & $\underline{24}$ & $\underline{65 \%}$ & $\underline{18}$ & $\underline{47 \%}$ & $\underline{7}$ & $\underline{50 \%}$ & $\underline{9}$ & $\underline{41 \%}$ & $\underline{8}$ & $33 \%$ & $\underline{13}$ & $\underline{41 \%}$ & $\underline{8}$ & $\underline{36 \%}$ \\
\hline & $\overline{100 \%}$ & 37 & $100 \%$ & 38 & $100 \%$ & 14 & $100 \%$ & 22 & $100 \%$ & 24 & $100 \%$ & 32 & $100 \%$ & 22 & $100 \%$ \\
\hline Total Number of Graduates & 427 & & 71 & & 74 & & 49 & & 58 & & 61 & & 63 & & 51 \\
\hline
\end{tabular}

\section{Maryland's 150-Hour Requirement (Table III and Table IV)}

The 150-hour requirement was adopted in Maryland in 1993, effective July 1, 1999. Since the 150-hour requirement had already been implemented before these interviews, the students were well aware of the necessity to enroll in an extra academic year of college in order to sit for the CPA Exam in Maryland. However, the remaining 30 credits beyond the typical 120-hour bachelor's degree do not have to result in a graduate degree. Courses may even be taken at a community college. The extra credits may be taken in a variety of ways and may be taken over any period of time. 
As shown in Table III, very few students (9\% overall and only 4\% of 2005-2006 graduates) were not planning to sit for the CPA exam. Six percent were planning to go to a State not requiring 150 hours. Thirty-eight percent of the graduates had already met the 150- hour requirement. Most of these students had the additional hours because they had changed majors or obtained a second bachelors degree. The others added a minor, a second major, or a concentration.

In 2006, Maryland's Higher Education Commission granted Towson the right to offer the MBA in conjunction with the University of Baltimore. As shown in Table IV, the newly formed joint UB/TU MBA program has not gone unnoticed by graduating TU accounting students, with $25 \%$ of the 2005-2006 students who were planning on graduate school opting to fulfill their 150 hours through this newly formed joint program. Another 13\% of that group stated that they would be enrolling in the UB/TU MS Accounting program, which is also in its infancy since its 2005 initiation. Twenty five percent were undecided as to how and where they would obtain the additional credits, while the remaining $37 \%$ planned to attend other graduate or post baccalaureate programs.

Table IV: Plans For Graduate School

\begin{tabular}{|c|c|c|c|c|c|c|c|c|c|c|c|c|c|c|c|}
\hline \multirow[t]{2}{*}{ Academic Year } & \multirow[b]{2}{*}{ Wt Avg } & \multicolumn{2}{|c|}{$2005-2006$} & \multicolumn{2}{|c|}{ 2004-2005 } & \multicolumn{2}{|c|}{ 2003-2004 } & \multicolumn{2}{|c|}{$2002-2003$} & \multicolumn{2}{|c|}{ 2001-2002 } & \multicolumn{2}{|c|}{$2000-2001$} & \multicolumn{2}{|c|}{ 1999-2000 } \\
\hline & & $\#$ & $\%$ & \# & $\%$ & $\#$ & $\%$ & $\#$ & $\%$ & $\#$ & $\%$ & $\#$ & $\%$ & $\#$ & $\%$ \\
\hline \multicolumn{16}{|l|}{ Graduate Schools \& Programs } \\
\hline Loyola MBA & $26 \%$ & 3 & $13 \%$ & 4 & $22 \%$ & & & 1 & $11 \%$ & 5 & $63 \%$ & 5 & $38 \%$ & 5 & $63 \%$ \\
\hline Joint TU/UB MS & $10 \%$ & 3 & $13 \%$ & 6 & $33 \%$ & & & & & & & & & & \\
\hline Joint TU/UB MBA & $7 \%$ & 6 & $25 \%$ & & & & & & & & & & & & \\
\hline UB MBA & $3 \%$ & & & 2 & $11 \%$ & & & & & & & 1 & $8 \%$ & & \\
\hline UB MS Tax & $2 \%$ & 1 & $4 \%$ & & & & & 1 & $11 \%$ & & & & & & \\
\hline TU IT & $2 \%$ & & & & & & & & & & & 2 & $15 \%$ & & \\
\hline Villa Julie Forensic & $3 \%$ & 1 & $4 \%$ & 2 & $11 \%$ & & & & & & & & & & \\
\hline Villa Julie IT & $1 \%$ & & & & & & & & & 1 & $13 \%$ & & & & \\
\hline UMUC MS & $2 \%$ & 2 & $8 \%$ & & & & & & & & & & & & \\
\hline Law School & $2 \%$ & 2 & $8 \%$ & & & & & & & & & & & & \\
\hline Howard Law/MBA & $1 \%$ & & & & & & & 1 & $11 \%$ & & & & & & \\
\hline JHU Post Bach & $1 \%$ & & & 1 & $6 \%$ & & & & & & & & & & \\
\hline Undecided & $\underline{37 \%}$ & $\underline{6}$ & $25 \%$ & $\underline{3}$ & $\underline{17 \%}$ & $\underline{7}$ & $\underline{100 \%}$ & $\underline{6}$ & $\underline{67 \%}$ & $\underline{2}$ & $\underline{25 \%}$ & $\underline{5}$ & $\underline{38 \%}$ & $\underline{3}$ & $\underline{38 \%}$ \\
\hline & $100 \%$ & & $100 \%$ & & $100 \%$ & & $100 \%$ & & $100 \%$ & & $100 \%$ & & $100 \%$ & & $100 \%$ \\
\hline Total Planning on Graduate School & 87 & 24 & & 18 & & 7 & & 9 & & 8 & & 13 & & 8 & \\
\hline \multicolumn{16}{|l|}{ Graduate Schools Summary } \\
\hline MBA & $38 \%$ & 9 & $38 \%$ & 6 & $33 \%$ & & & 2 & $22 \%$ & 5 & $63 \%$ & 6 & $46 \%$ & 5 & $63 \%$ \\
\hline Spec MS, Law \& Post-bach & $25 \%$ & 9 & $38 \%$ & 9 & $50 \%$ & & & 1 & $11 \%$ & 1 & $13 \%$ & 2 & $15 \%$ & & \\
\hline Undecided & $\underline{37 \%}$ & $\underline{6}$ & $\underline{25 \%}$ & $\underline{3}$ & $\underline{17 \%}$ & $\underline{7}$ & $\underline{100 \%}$ & $\underline{6}$ & $67 \%$ & $\underline{2}$ & $25 \%$ & $\underline{5}$ & $\underline{38 \%}$ & $\underline{3}$ & $\underline{38 \%}$ \\
\hline & $\overline{100 \%}$ & & $\overline{100 \%}$ & & $\overline{100 \%}$ & & $\overline{100 \%}$ & & $100 \%$ & & $100 \%$ & & $\overline{100 \%}$ & & $100 \%$ \\
\hline Total Planning on Graduate School & 87 & 24 & & 18 & & 7 & & 9 & & 8 & & 13 & & 8 & \\
\hline
\end{tabular}

\section{Accounting Exit Exam (Table V)}

In order to graduate with a major in accounting, students were initially required to take and pass the AICPA's Level II test. When the AICPA dropped the Level II exam in the 1980s, a similar, nationally normed test known as the Achievement Test for Accounting Graduates (ATAG), developed by the Psychological Corporation, took its place as one of the requirements for graduation as an accounting major. Nichols (1991) suggests that the ATAG is most appropriate for assessing accounting majors at four-year schools while Herring and Izard (1992) confirm the ATAG is useful for measuring technical accounting skills (Stivers, Campbell and Hermanson, 2000).

The ATAG covers five specific areas (accounting information systems, auditing, cost and managerial, financial, and taxation), which correspond with courses in our accounting curriculum. Test booklets can be reused and the tests are scored at TU. The test was offered three times per year. The minimum-passing percentile for TU accounting majors was $50 \%$. 
As shown in Table $\mathrm{V}$, over the six-year exit interview period nearly half of the students passed on the first attempt, and $78 \%$ passed the test on the first or second attempt. The first and second attempt pass rates are downward biased since students may take this test prior to completing all of their accounting coursework and they often take it early to "see what it's like." Ultimately, almost everyone passed the ATAG, but for the few who did not pass an accounting degree was not awarded.

Table V: Accounting Exit Exam

\begin{tabular}{|c|c|c|c|c|c|c|c|c|c|c|c|c|c|c|c|}
\hline \multirow[t]{2}{*}{ Academic Year } & \multirow[b]{2}{*}{ Wt Avg } & \multicolumn{2}{|c|}{ 2005-2006 } & \multicolumn{2}{|c|}{ 2004-2005 } & \multicolumn{2}{|c|}{ 2003-2004 } & \multicolumn{2}{|c|}{$2002-2003$} & \multicolumn{2}{|c|}{ 2001-2002 } & \multicolumn{2}{|c|}{$2000-2001$} & \multicolumn{2}{|c|}{$1999-2000$} \\
\hline & & \# & $\%$ & $\#$ & $\%$ & $\#$ & $\%$ & \# & $\%$ & $\#$ & $\%$ & $\#$ & $\%$ & $\#$ & $\%$ \\
\hline \multicolumn{16}{|l|}{ Test Attempts } \\
\hline One & $48.7 \%$ & 33 & $46 \%$ & 36 & $49 \%$ & 29 & $59 \%$ & 27 & $47 \%$ & 31 & $51 \%$ & 28 & $44 \%$ & 24 & $47 \%$ \\
\hline Two & $29.0 \%$ & 23 & $32 \%$ & 25 & $34 \%$ & 12 & $24 \%$ & 13 & $22 \%$ & 15 & $25 \%$ & 20 & $32 \%$ & 16 & $31 \%$ \\
\hline Three & $14.8 \%$ & 9 & $13 \%$ & 9 & $12 \%$ & 7 & $14 \%$ & 10 & $17 \%$ & 9 & $15 \%$ & 12 & $19 \%$ & 7 & $14 \%$ \\
\hline Four & $5.2 \%$ & 5 & $7 \%$ & 2 & $3 \%$ & 1 & $2 \%$ & 6 & $10 \%$ & 2 & $3 \%$ & 3 & $5 \%$ & 3 & $6 \%$ \\
\hline Five & $2.1 \%$ & 1 & $1 \%$ & 1 & $1 \%$ & & & 2 & $3 \%$ & 4 & $7 \%$ & & & 1 & $2 \%$ \\
\hline \multirow[t]{2}{*}{ Six } & $\underline{0.2 \%}$ & & & 1 & $1 \%$ & & & & & & & & & & \\
\hline & $100.0 \%$ & 71 & $100 \%$ & 74 & $100 \%$ & 49 & $100 \%$ & 58 & $100 \%$ & 61 & $100 \%$ & 63 & $100 \%$ & 51 & $100 \%$ \\
\hline Total \# of Grads & 427 & & 71 & & 74 & & 49 & & 58 & & 61 & & 63 & & 51 \\
\hline
\end{tabular}

\begin{tabular}{|c|c|c|c|c|c|c|c|c|c|c|c|c|c|c|c|}
\hline \multirow{3}{*}{ Academic Year } & \multirow[b]{3}{*}{ Wt Avg } & \multirow{2}{*}{\multicolumn{2}{|c|}{\begin{tabular}{|l|}
$2005-2006$ \\
\end{tabular}}} & \multirow{2}{*}{\multicolumn{2}{|c|}{ 2004-2005 }} & \multirow{2}{*}{\multicolumn{2}{|c|}{ 2003-2004 }} & \multirow{2}{*}{\multicolumn{2}{|c|}{$2002-2003$}} & \multirow{2}{*}{\multicolumn{2}{|c|}{ 2001-2002 }} & \multirow{2}{*}{\multicolumn{2}{|c|}{$2000-2001$}} & \multirow{2}{*}{\multicolumn{2}{|c|}{ 1999-2000 }} \\
\hline & & & & & & & & & & & & & & & \\
\hline & & $\#$ & $\%$ & \# & $\%$ & $\#$ & $\%$ & \# & $\%$ & $\#$ & $\%$ & \# & $\%$ & \# & $\%$ \\
\hline \multicolumn{16}{|l|}{ Job Placement Data } \\
\hline Big 4 Firms (Big 5 pre 2002) & $19 \%$ & 11 & $15 \%$ & 14 & $19 \%$ & 10 & $20 \%$ & 10 & $17 \%$ & 10 & $16 \%$ & 17 & $27 \%$ & 10 & $20 \%$ \\
\hline Other Public Accounting Firms & $37 \%$ & 27 & $38 \%$ & 34 & $46 \%$ & 19 & $39 \%$ & 12 & $21 \%$ & 31 & $51 \%$ & 18 & $29 \%$ & 17 & $33 \%$ \\
\hline Corporate Accounting & $11 \%$ & 6 & $8 \%$ & 7 & $9 \%$ & 8 & $16 \%$ & 7 & $12 \%$ & 6 & $10 \%$ & 5 & $8 \%$ & 8 & $16 \%$ \\
\hline Financial Services & $5 \%$ & 5 & $7 \%$ & 1 & $1 \%$ & 4 & $8 \%$ & 4 & $7 \%$ & & & 4 & $6 \%$ & 4 & $8 \%$ \\
\hline Not for Profit & $2 \%$ & & & 4 & $5 \%$ & 1 & $2 \%$ & 3 & $5 \%$ & & & & & & \\
\hline Government & $6 \%$ & 5 & $7 \%$ & 4 & $5 \%$ & 1 & $2 \%$ & 3 & $5 \%$ & 3 & $5 \%$ & 5 & $8 \%$ & 4 & $8 \%$ \\
\hline Non-Accounting & $1 \%$ & 1 & $1 \%$ & & & & & & & & & 3 & $5 \%$ & 1 & $2 \%$ \\
\hline Military & $0 \%$ & & & & & & & 1 & $2 \%$ & & & & & & \\
\hline Graduate School & $3 \%$ & 4 & $6 \%$ & 3 & $4 \%$ & & & 2 & $3 \%$ & 1 & $2 \%$ & 2 & $3 \%$ & 2 & $4 \%$ \\
\hline Undecided & $15 \%$ & 12 & $\underline{17 \%}$ & $\underline{7}$ & $\underline{9 \%}$ & $\underline{6}$ & $\underline{12 \%}$ & $\underline{16}$ & $\underline{28 \%}$ & $\underline{10}$ & $\underline{16 \%}$ & $\underline{9}$ & $\underline{14 \%}$ & $\underline{5}$ & $\underline{10 \%}$ \\
\hline & $\overline{100 \%}$ & $\overline{71}$ & $\overline{100 \%}$ & $\overline{74}$ & $100 \%$ & 49 & $\overline{100 \%}$ & $\overline{58}$ & $\overline{100 \%}$ & $\overline{61}$ & $\overline{100 \%}$ & 63 & $\overline{100 \%}$ & $\overline{51}$ & $\overline{100 \%}$ \\
\hline \multicolumn{16}{|l|}{$\begin{array}{l}\text { Audit/Tax Breakdown } \\
\text { Big } 4 \text { Firms (Big } 5 \text { pre 2002) }\end{array}$} \\
\hline Audit & $84 \%$ & 9 & $82 \%$ & 12 & $86 \%$ & 9 & $90 \%$ & 8 & $80 \%$ & 10 & $100 \%$ & 13 & $76 \%$ & 8 & $80 \%$ \\
\hline $\begin{array}{l}\text { Tax } \\
\text { Other Public Accounting Firms }\end{array}$ & $16 \%$ & 2 & $18 \%$ & 2 & $14 \%$ & 1 & $10 \%$ & 2 & $20 \%$ & & & 4 & $24 \%$ & 2 & $20 \%$ \\
\hline Audit & $87 \%$ & 26 & $96 \%$ & 29 & $85 \%$ & 18 & $95 \%$ & 12 & $100 \%$ & 27 & $87 \%$ & 14 & $78 \%$ & 12 & $71 \%$ \\
\hline Tax & $13 \%$ & 1 & $4 \%$ & 5 & $15 \%$ & 1 & $5 \%$ & & & 4 & $13 \%$ & 4 & $22 \%$ & 5 & $29 \%$ \\
\hline
\end{tabular}

\section{Job Placement (Table VI and Table VII)}

Table VI summarizes job placement data for the graduating seniors. In addition to the services offered by the university placement office, the Accounting Department supports job placement through an "Accounting Open House" each fall that attracts over 60 potential employers. At this event, students are encouraged to "dress for success", bring multiple copies of their resumes, and to chat with potential employers in an informal setting. Upperclassmen attend to seek permanent employment or an internship prior to the senior year, while underclassmen are also encouraged to attend to seek inroads into possible internships. 
Table VII: Placement Determinants

Academic Year

\begin{tabular}{|l|l|l|l|l|l|l|}
\hline 2005-2006 & 2004-2005 & 2003-2004 & 2002-2003 & 2001-2002 & 2000-2001 & 1999-2000 \\
\hline
\end{tabular}

\begin{tabular}{|c|c|c|c|c|c|c|c|c|c|c|c|c|c|c|c|}
\hline & Wt Avg & $\#$ & $\%$ & \# & $\%$ & \# & $\%$ & $\#$ & $\%$ & $\#$ & $\%$ & $\#$ & $\%$ & $\#$ & $\%$ \\
\hline \multicolumn{16}{|l|}{ How Placement Was Obtained } \\
\hline Junior Year Interviews & $2 \%$ & 2 & $3 \%$ & 3 & $4 \%$ & & & & & 1 & $2 \%$ & & & & \\
\hline Fall of Senior Year Interviews & $25 \%$ & 15 & $21 \%$ & 28 & $38 \%$ & 15 & $31 \%$ & 11 & $19 \%$ & 10 & $16 \%$ & & & & \\
\hline Spring of Senior Year Interviews & $6 \%$ & 7 & $10 \%$ & 4 & $5 \%$ & 2 & $4 \%$ & 5 & $9 \%$ & & & & & & \\
\hline Internship led to Full Time Position & $30 \%$ & 21 & $30 \%$ & 16 & $22 \%$ & 13 & $27 \%$ & 14 & $24 \%$ & 30 & $49 \%$ & & & & \\
\hline Stayed with Current Full Time Employer & $12 \%$ & 6 & $8 \%$ & 8 & $11 \%$ & 9 & $18 \%$ & 8 & $14 \%$ & 7 & $11 \%$ & & & & \\
\hline Self-initiated job search & $\mathbf{5 \%}$ & 4 & $6 \%$ & 5 & $7 \%$ & 4 & $8 \%$ & 1 & $2 \%$ & 2 & $3 \%$ & & & & \\
\hline No Placement & $\underline{20 \%}$ & 16 & $\underline{23 \%}$ & 10 & $\underline{14 \%}$ & $\underline{6}$ & $12 \%$ & 19 & $\underline{33 \%}$ & $\underline{11}$ & $\underline{18 \%}$ & & & & \\
\hline & $\overline{100 \%}$ & $\overline{71}$ & $\overline{100 \%}$ & $\overline{74}$ & $\overline{100 \%}$ & 49 & $\overline{100 \%}$ & $\overline{58}$ & $\overline{100 \%}$ & $\overline{61}$ & $\overline{100 \%}$ & & & & \\
\hline \multicolumn{16}{|l|}{ When Placement Was Decided } \\
\hline Junior Year & $30 \%$ & 23 & $32 \%$ & 22 & $30 \%$ & 19 & $39 \%$ & 10 & $17 \%$ & 21 & $34 \%$ & & & & \\
\hline Fall Semester of Senior Year & $34 \%$ & 20 & $28 \%$ & 37 & $50 \%$ & 16 & $33 \%$ & 20 & $34 \%$ & 14 & $23 \%$ & & & & \\
\hline Spring Semester of Senior Year & $16 \%$ & 12 & $17 \%$ & 5 & $7 \%$ & 8 & $16 \%$ & 9 & $16 \%$ & 15 & $25 \%$ & & & & \\
\hline No Placement at Graduation & $\underline{20 \%}$ & $\underline{16}$ & $\underline{23 \%}$ & $\underline{10}$ & $\underline{14 \%}$ & $\underline{6}$ & $\underline{12 \%}$ & $\underline{19}$ & $\underline{33 \%}$ & $\underline{11}$ & $\underline{18 \%}$ & & & & \\
\hline & $\overline{100 \%}$ & $\overline{71}$ & $\overline{100 \%}$ & $\overline{74}$ & $\overline{100 \%}$ & $\overline{49}$ & $\overline{100 \%}$ & $\overline{58}$ & $\overline{100 \%}$ & $\overline{61}$ & $\overline{100 \%}$ & & & & \\
\hline \multicolumn{16}{|c|}{ Internship Exp While in School } \\
\hline Three Internships & $3 \%$ & 2 & $3 \%$ & 2 & $3 \%$ & 1 & $2 \%$ & 2 & $3 \%$ & 1 & $2 \%$ & 1 & $2 \%$ & 2 & $4 \%$ \\
\hline Two Internships & $17 \%$ & 15 & $21 \%$ & 15 & $20 \%$ & 9 & $18 \%$ & 5 & $9 \%$ & 12 & $20 \%$ & 12 & $19 \%$ & 6 & $12 \%$ \\
\hline One Internship & $41 \%$ & 24 & $34 \%$ & 33 & $45 \%$ & 19 & $39 \%$ & 21 & $36 \%$ & 30 & $49 \%$ & 27 & $43 \%$ & 20 & $39 \%$ \\
\hline None - working Full-time & $14 \%$ & 10 & $14 \%$ & 12 & $16 \%$ & 4 & $8 \%$ & 13 & $22 \%$ & 9 & $15 \%$ & 3 & $5 \%$ & 8 & $16 \%$ \\
\hline None - working Part-time & $16 \%$ & 11 & $15 \%$ & 5 & $7 \%$ & 11 & $22 \%$ & 11 & $19 \%$ & 6 & $10 \%$ & 13 & $21 \%$ & 11 & $22 \%$ \\
\hline None - not working & $\underline{10 \%}$ & $\underline{9}$ & $\underline{13 \%}$ & 1 & $9 \%$ & $\underline{5}$ & $\underline{10 \%}$ & $\underline{6}$ & $\underline{10 \%}$ & $\underline{3}$ & $\underline{5 \%}$ & $\underline{7}$ & $\underline{11 \%}$ & $\underline{4}$ & $\underline{8 \%}$ \\
\hline \multicolumn{15}{|l|}{ Internship Employer } & $100 \%$ \\
\hline Big 4 Firms (Big 5 pre 2002) & $15 \%$ & 7 & $12 \%$ & 7 & $10 \%$ & 8 & $20 \%$ & 8 & $22 \%$ & 10 & $18 \%$ & 7 & $13 \%$ & 5 & $13 \%$ \\
\hline Other Public Acc & $51 \%$ & 31 & $52 \%$ & 40 & $58 \%$ & 18 & $45 \%$ & 15 & $41 \%$ & 36 & $63 \%$ & 22 & $41 \%$ & 20 & $53 \%$ \\
\hline Corporate Accounting & $12 \%$ & 5 & $8 \%$ & 4 & $6 \%$ & 10 & $25 \%$ & 7 & $19 \%$ & 4 & $7 \%$ & 11 & $20 \%$ & 2 & $5 \%$ \\
\hline Financial Services & $8 \%$ & 7 & $12 \%$ & 9 & $13 \%$ & 2 & $5 \%$ & 2 & $5 \%$ & 4 & $7 \%$ & 1 & $2 \%$ & 3 & $8 \%$ \\
\hline Government & $5 \%$ & 3 & $5 \%$ & 3 & $4 \%$ & 1 & $3 \%$ & 3 & $8 \%$ & & & 5 & $9 \%$ & 3 & $8 \%$ \\
\hline Not for Profit & $6 \%$ & 5 & $8 \%$ & 6 & $9 \%$ & 1 & $3 \%$ & 2 & $5 \%$ & 2 & $4 \%$ & 1 & $2 \%$ & 5 & $13 \%$ \\
\hline Non Accounting & $\underline{3 \%}$ & $\underline{2}$ & $\underline{3 \%}$ & & & & & & & $\underline{1}$ & $\underline{2 \%}$ & $\underline{7}$ & $\underline{13 \%}$ & & \\
\hline & $\overline{100 \%}$ & 60 & $100 \%$ & 69 & $100 \%$ & 40 & $100 \%$ & 37 & $100 \%$ & 57 & $100 \%$ & 54 & $\overline{100 \%}$ & 38 & $100 \%$ \\
\hline \multicolumn{16}{|l|}{ Full-time or Part-time Employer } \\
\hline Big 4 Firms (Big 5 pre 2002) & $\mathbf{0 \%}$ & & & & & & & & & & & & $0 \%$ & & $0 \%$ \\
\hline Other Public Accounting Firms & $14 \%$ & 2 & $10 \%$ & 2 & $11 \%$ & 2 & $11 \%$ & 3 & $12 \%$ & 3 & $19 \%$ & 3 & $17 \%$ & 5 & $25 \%$ \\
\hline Corporate Accounting & $37 \%$ & 6 & $29 \%$ & 10 & $53 \%$ & 5 & $26 \%$ & 10 & $40 \%$ & 6 & $38 \%$ & 6 & $33 \%$ & 8 & $40 \%$ \\
\hline Financial Services & $20 \%$ & 4 & $19 \%$ & 2 & $11 \%$ & 5 & $26 \%$ & 4 & $16 \%$ & 5 & $31 \%$ & 5 & $28 \%$ & 3 & $15 \%$ \\
\hline Small Business & $6 \%$ & 5 & $24 \%$ & 1 & $5 \%$ & 1 & $5 \%$ & & & & & & & 1 & $5 \%$ \\
\hline Not for Profit & $9 \%$ & 2 & $10 \%$ & 3 & $16 \%$ & 5 & $26 \%$ & & & 1 & $6 \%$ & & & 1 & $5 \%$ \\
\hline Government & $5 \%$ & 2 & $10 \%$ & 1 & $5 \%$ & 1 & $5 \%$ & & & 1 & $6 \%$ & 1 & $6 \%$ & 1 & $5 \%$ \\
\hline Own Business & $1 \%$ & & & & & & & 2 & $8 \%$ & & & & & & $0 \%$ \\
\hline \multirow[t]{2}{*}{ Non-Accounting } & $\underline{7 \%}$ & & & & & & & $\underline{6}$ & $24 \%$ & & & $\underline{3}$ & $17 \%$ & 1 & $5 \%$ \\
\hline & $\overline{100 \%}$ & 21 & $100 \%$ & 19 & $100 \%$ & 19 & $100 \%$ & 25 & $\overline{100 \%}$ & 16 & $100 \%$ & $\overline{18}$ & $\overline{100 \%}$ & 20 & $100 \%$ \\
\hline Total Number of Graduates & 427 & & 71 & & 74 & & 49 & & 58 & & 61 & & 63 & & 51 \\
\hline
\end{tabular}

The employment position was obtained in various ways, such as, interviews, internships, self-initiated searches, and staying on with their present full time employer. However, as shown in Table VII, the greatest number of successful placements was through on-campus interviews and student internships. Many internships resulted in permanent placement being accepted in the student's junior year. Even though some students had multiple internships, $41 \%$ held only one internship position. Only 5\% of the graduates obtained their positions through self-initiated job searches. 


\section{Perceived Program Strengths and Weaknesses (Table VIII and Table IX)}

During the interview process, the students were asked what they liked and what they disliked about their experience as Towson accounting majors. This elicited 1,338 total comments (926 positive and 412 negative) from the 427 graduates. These comments have been sorted into six categories: overall program satisfaction/dissatisfaction, program characteristics, curriculum, specific courses, course characteristics, and administration.

As shown in Table VIII, 55\% of the students made a comment of general overall satisfaction with the program and over 9\% commented that the Accounting Department is either the best department within the TU College of Business or the best department of multiple colleges or universities that they had attended. As shown in Table IX, there were no general comments of overall dissatisfaction with the program, but many complaints within the specific categories.

Regarding characteristics of the accounting program, $64 \%$ of graduating seniors cited their general satisfaction with the professors and $26 \%$ indicated satisfaction with the challenging nature of the program. There were also a large number of positive comments about specific aspects of the program. Unfortunately, but not too surprisingly, the very aspects that pleased some graduates displeased others. While $64 \%$ cited satisfaction with professors, $21 \%$ had complaints about specific professors. While $7 \%$ mentioned the focus on the CPA Exam and 5\% noted Career Focus and Guidance positively, 3\% referred to the program as Too Academic/not practical enough. While 3\% cited the focus on the APAT and ATAG positively, 3\% mentioned that the APAT was unnecessary and $15 \%$ cited the ATAG as unnecessary and stressful. While 3\% commented positively on course availability, $5 \%$ had complaints about class scheduling and availability. Curriculum advising was noted positively by $2 \%$, but negatively by $5 \%$.

Very few students made comments that we classified in the other categories. Among the comments under curriculum, there were complaints of unnecessary classes and of the need for additional classes. Several specific courses were cited both positively and negatively, but there was more dissatisfaction mentioned regarding the Business Ethics class, the Tax class, the Cornerstone class (a business school requirement) and Management classes in general. . In terms of administration, the accounting office staff was recognized as a plus and the need for more technology and technological support was a complaint.

\section{CONCLUSION}

The exit interviews have served to assist the Department of Accounting in evaluating its performance as judged by the students served. The positive comments assist to reinforce the quality activities of the department, while the negative comments permit the department to become aware of areas of perceived weakness that are in need of improvement. By receiving annual feedback from a valuable, relevant source, the department is able to continuously monitor, adjust, and improve its performance.

Towson University (TU) requires each professor to complete an annual report setting out what the professor plans to accomplish in the upcoming academic period and the extent to which the professor has met similar goals of the preceding academic year. The report emphasizes a balance among three duties: teaching, research, and service. Since TU places a significant amount of emphasis on teaching, teaching is weighted the greatest of the three (at least $70 \%$ ). Therefore, student complaints regarding teaching inadequacies are of major concern. Individual student complaints regarding specific professors or specific courses are addressed by the chairperson through discussion with the professor, and are usually easily resolved. The professor himself echoed complaints about the textbook for the tax class, for instance, and the text was changed. A pattern of complaints regarding the classroom abilities of a professor resulted in a mentoring program by peer professors in order to improve classroom performance. 
Table VIII: Positive Comments

Academic Year

Overall Program Satisfaction

Overall Satisfaction of Program

Best Department in CBE

Best of Multiple Schools Attended $\mathbf{3 \%}$

Program Characteristics

Professors/Real World Professors

Challenging Program

Focus on CPA Exam

Beta Alpha Psi

Career Focus and Guidance

Program Leadership

Networking

Internships

Focus on APAT \& ATAG

Course Availability

Small Classes

Curriculum Advising

Camaraderie, environment

Interested in Students/Stu Focus

Accreditation of Program

KSA's

Other(study abroad,tech,diversity)

\section{Curriculum}

Real World curriculum

Overall Accounting Curriculum

Three Semester Intermediate

Accounting Electives

120 hour program

Specific Courses

Accounting Principles

Auditing

Other (7 different courses)

Course Characteristics

Practical Assignments

Case Studies

Other (teams, asssignments, texts)

Administration

Department Office Staff

CBE Student Center

Other(career support,class locat'n)

total

Comments per Student

Total Number of Graduates

$6 \%$

$26 \%$

$7 \%$

$5 \%$

$5 \%$

$5 \%$

$4 \%$

$\mathbf{3 \%}$

$3 \%$

$3 \%$

$2 \%$

$2 \%$

$1 \%$

$1 \%$

$1 \%$

$1 \%$

$3 \%$

$2 \%$

$1 \%$

$1 \%$

$0 \%$

$2 \%$

$1 \%$

$1 \%$

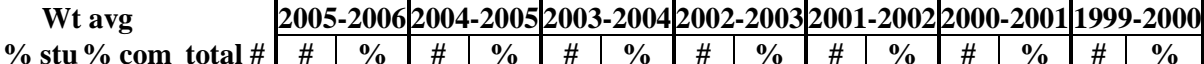

\begin{tabular}{ll|ll|lllllllllllll}
$\mathbf{5 5 \%}$ & $\mathbf{2 3 5}$ & 35 & $49 \%$ & 49 & $66 \%$ & 37 & $76 \%$ & 37 & $64 \%$ & 33 & $54 \%$ & 31 & $49 \%$ & 13 & $25 \%$
\end{tabular}

$\mathbf{3 0 \%}$

27

\begin{tabular}{ll|ll|ll}
7 & $10 \%$ & 4 & $5 \%$ & 1 & $2 \%$
\end{tabular}

\begin{tabular}{ll|ll}
4 & $7 \%$ & 3 & $5 \%$
\end{tabular}

$6 \quad 10 \%$

$24 \%$

\begin{tabular}{l|l|ll}
12 & \\
274
\end{tabular}

$510 \%$

$64 \%$

$1 \%$

(1)

\begin{tabular}{l|ll|ll|l|l}
274 & & & \\
274 & 38 & $54 \%$ & 54 & $73 \%$ & 35 & $71 \%$
\end{tabular}

\begin{tabular}{l|ll|ll|ll}
113 & 26 & $37 \%$ & 20 & $27 \%$ & 18 & $37 \%$
\end{tabular}

\begin{tabular}{l|ll|ll}
28 & 8 & $11 \%$ & 9 & $12 \%$
\end{tabular}

\begin{tabular}{l|ll|ll}
23 & 1 & $1 \%$ & 5 & $7 \%$
\end{tabular}

$\begin{array}{lllllllll}4 \% & 6 & 10 \% & 5 & 8 \% & 3 & 5 \% & 1 & 2 \%\end{array}$

\begin{tabular}{l|llllllllllllll}
23 & 4 & $6 \%$ & 6 & $8 \%$ & 3 & $6 \%$ & 3 & $5 \%$ & 4 & $7 \%$ & 2 & $3 \%$ & 1 & $2 \%$
\end{tabular}

\begin{tabular}{l|ll|ll|ll|ll|ll|ll|ll}
20 & 1 & $1 \%$ & 2 & $3 \%$ & 4 & $8 \%$ & 2 & $3 \%$ & 4 & $7 \%$ & 4 & $6 \%$ & 3 & $6 \%$
\end{tabular}

19

14

12

\begin{tabular}{llll|ll}
7 & $10 \%$ & 8 & $11 \%$ & 1 & $2 \%$
\end{tabular}

$\begin{array}{ll}5 & 7 \% \\ 3 & 4 \%\end{array}$

\begin{tabular}{ll|ll|ll|ll}
1 & $1 \%$ & 3 & $4 \%$ & 1 & $2 \%$ & 2 & $3 \%$
\end{tabular}

10

7

5

5

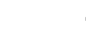

$62 \%$

$3 \%$

$\begin{array}{lll}2 & 3 \%\end{array}$

\begin{tabular}{l|ll|ll}
$\underline{\mathbf{5}}_{\mathbf{5 7 6}}$ & 1 & $1 \%$ & 1 & $1 \%$
\end{tabular}

(11

11
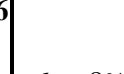

$$
\begin{array}{c|cc|cc|cc}
11 & 6 & 8 \% & 2 & 3 \% & 3 & 6 \% \\
9 & 1 & 1 \% & & & &
\end{array}
$$$$
\begin{array}{l|ll|ll}
5 & 2 & 3 \% & 1 & 1 \%
\end{array}
$$

4

31

$1 \%$

$1 \%$

$2 \%$

17

$\%$

0\%

3

\begin{tabular}{lll|ll|ll|} 
& $\mathbf{3}$ & & & & & \\
& $\mathbf{2}$ & & & & 1 & $1 \%$ \\
& $\underline{3}$ & & 1 & $1 \%$ & & \\
$1 \%$ & & $\mathbf{8}$ & &
\end{tabular}

15

8 
Table IX: Negative Comments

\begin{tabular}{|c|c|c|c|c|c|c|c|c|c|c|c|c|c|c|c|c|c|}
\hline \multirow[t]{2}{*}{ Academic Year } & \multirow{2}{*}{\multicolumn{3}{|c|}{$\begin{array}{c}\text { Wt avg } \\
\text { \% stu \% com total \# }\end{array}$}} & \multicolumn{2}{|c|}{$2005-2006$} & \multicolumn{2}{|c|}{$2004-2005$} & \multicolumn{2}{|c|}{ 2003-2004 } & \multicolumn{2}{|c|}{$2002-2003$} & \multicolumn{2}{|c|}{ 2001-2002 } & \multicolumn{2}{|c|}{ 2000-2001 } & \multicolumn{2}{|c|}{ 1999-2000 } \\
\hline & & & & $\#$ & $\%$ & $\#$ & $\%$ & $\#$ & $\%$ & $\#$ & $\%$ & $\#$ & $\%$ & \# & $\%$ & $\#$ & $\%$ \\
\hline Overall Program Dissatisfaction & $\mathbf{0 \%}$ & & $\mathbf{0}$ & 0 & $0 \%$ & 0 & $0 \%$ & 0 & $0 \%$ & 0 & $0 \%$ & 0 & $0 \%$ & 0 & $0 \%$ & 0 & $0 \%$ \\
\hline \multicolumn{18}{|l|}{ Program Characteristics } \\
\hline Specific Professor Complaints & $21 \%$ & & 89 & 18 & $25 \%$ & 13 & $18 \%$ & 10 & $20 \%$ & 15 & $26 \%$ & 9 & $15 \%$ & 10 & $16 \%$ & 14 & $27 \%$ \\
\hline ATAG is unnecessary and stressful & $15 \%$ & & 63 & 13 & $18 \%$ & 6 & $8 \%$ & 9 & $18 \%$ & 9 & $16 \%$ & 6 & $10 \%$ & 14 & $22 \%$ & 6 & $12 \%$ \\
\hline Advising & $5 \%$ & & 22 & 1 & $1 \%$ & 3 & $4 \%$ & & & 1 & $2 \%$ & 5 & $8 \%$ & 6 & $10 \%$ & 6 & $12 \%$ \\
\hline Class Scheduling \& Availability & $5 \%$ & & 22 & 3 & $4 \%$ & 4 & $5 \%$ & 1 & $2 \%$ & 4 & $7 \%$ & 3 & $5 \%$ & 1 & $2 \%$ & 6 & $12 \%$ \\
\hline Too Academic/not practical enough & $3 \%$ & & 14 & 1 & $1 \%$ & 1 & $1 \%$ & & & & & 3 & $5 \%$ & 3 & $5 \%$ & 6 & $12 \%$ \\
\hline APAT is unnecessary & $3 \%$ & & 11 & & & & & & & 1 & $2 \%$ & 6 & $10 \%$ & 3 & $5 \%$ & 1 & $2 \%$ \\
\hline Too much CPA emphasis & $1 \%$ & & 6 & & & 1 & $1 \%$ & & & & & 1 & $2 \%$ & 2 & $3 \%$ & 2 & $4 \%$ \\
\hline More CPA exam information & $1 \%$ & & 3 & 1 & $1 \%$ & & & & & & & 1 & $2 \%$ & 1 & $2 \%$ & & \\
\hline Nonstandard Grading & $1 \%$ & & 3 & & & 1 & $1 \%$ & 1 & $2 \%$ & & & & & & & 1 & $2 \%$ \\
\hline Team Teaching & $1 \%$ & & 3 & & & & & & & & & & & 2 & $3 \%$ & 1 & $2 \%$ \\
\hline Class Too Large & $0 \%$ & & 2 & & & & & & & 1 & $2 \%$ & & & & & 1 & $2 \%$ \\
\hline More Recruiting Activities & $\mathbf{0 \%}$ & & 2 & & & & & 1 & $2 \%$ & 1 & $2 \%$ & & & & & & \\
\hline Quality of Evening Classes & $0 \%$ & & 2 & & & & & & & & & 1 & $2 \%$ & & & 1 & $2 \%$ \\
\hline Towson University/CBE too distant & $0 \%$ & & 2 & & & & & & & & & & & & & 2 & $4 \%$ \\
\hline Other (6 miscellaneous comments) & $1 \%$ & & $\underline{6}$ & & & 2 & $3 \%$ & 1 & $2 \%$ & 2 & $3 \%$ & 1 & $2 \%$ & & & & \\
\hline & & $61 \%$ & 25 & & & & & & & & & & & & & & \\
\hline \multicolumn{18}{|l|}{ Curriculum } \\
\hline Unnecessary classes outside accounting & $4 \%$ & & 19 & & & & & & & & & 12 & $20 \%$ & 7 & $11 \%$ & & \\
\hline Need a Graduate Program / 150 hr & $2 \%$ & & 7 & & & & & & & 1 & $2 \%$ & & & 3 & $5 \%$ & 3 & $6 \%$ \\
\hline Require Internship & $1 \%$ & & 4 & & & 1 & $1 \%$ & & & & & & & 1 & $2 \%$ & 2 & $4 \%$ \\
\hline Need Principles for Acctg Majors & $1 \%$ & & 3 & & & & & & & & & 1 & $2 \%$ & 2 & $3 \%$ & & \\
\hline More required classes & $1 \%$ & & 3 & 1 & $1 \%$ & 1 & $1 \%$ & & & & & 1 & $2 \%$ & & & & \\
\hline Other (10 miscellaneous comments) & $3 \%$ & & $\underline{12}$ & 1 & $2 \%$ & 1 & $1 \%$ & & & 5 & $9 \%$ & 1 & $2 \%$ & 1 & $2 \%$ & 3 & $6 \%$ \\
\hline & & $12 \%$ & 48 & & & & & & & & & & & & & & \\
\hline \multicolumn{18}{|l|}{ Specific Courses } \\
\hline Business Ethics class & $3 \%$ & & 14 & & & 4 & $5 \%$ & 6 & $12 \%$ & 4 & $7 \%$ & & & & & & \\
\hline Tax classes and book & $2 \%$ & & 9 & 2 & $3 \%$ & 1 & $1 \%$ & 3 & $6 \%$ & 3 & $5 \%$ & & & & & & \\
\hline Cornerstone class & $2 \%$ & & 7 & 3 & $4 \%$ & 1 & $1 \%$ & & & 3 & $5 \%$ & & & & & & \\
\hline Management classes & $2 \%$ & & 7 & & & 5 & $7 \%$ & 2 & $4 \%$ & & & & & & & & \\
\hline Accounting Principles class & $1 \%$ & & 6 & 4 & $6 \%$ & 1 & $1 \%$ & & & & & & & & & 1 & $2 \%$ \\
\hline Other (6 different courses) & $2 \%$ & & $\underline{9}$ & 5 & $7 \%$ & 2 & $3 \%$ & & & 1 & $2 \%$ & 1 & $2 \%$ & & & & \\
\hline & & $13 \%$ & 52 & & & & & & & & & & & & & & \\
\hline \multicolumn{18}{|l|}{ Course Characteristics } \\
\hline Return Exams & $1 \%$ & & 3 & 2 & $3 \%$ & & & 1 & $2 \%$ & & & & & & & & \\
\hline Too much group work & $1 \%$ & & 3 & & & & & 1 & $2 \%$ & & & & & & & 2 & $4 \%$ \\
\hline Too much test emphasis & $1 \%$ & & 3 & & & & & & & & & & & 3 & $5 \%$ & & \\
\hline Other (6 miscellaneous comments) & $2 \%$ & & $\underline{7}$ & 1 & $1 \%$ & & & 1 & $2 \%$ & 2 & $3 \%$ & 1 & $2 \%$ & 1 & $2 \%$ & 1 & $2 \%$ \\
\hline & & $4 \%$ & 16 & & & & & & & & & & & & & & \\
\hline Administration & & & & & & & & & & & & & & & & & \\
\hline More Technology \& Support & $6 \%$ & & 26 & 1 & $1 \%$ & 4 & $5 \%$ & 1 & $2 \%$ & & & 8 & $13 \%$ & 5 & $8 \%$ & 7 & $14 \%$ \\
\hline Promotion of Program Activities & $1 \%$ & & 5 & & & & & 2 & $4 \%$ & 2 & $3 \%$ & & & & & 1 & $2 \%$ \\
\hline Need Student Newsletter & $1 \%$ & & 3 & & & & & & & & & 1 & $2 \%$ & 2 & $3 \%$ & & \\
\hline Better Publicity of Program & $0 \%$ & & 2 & & & & & & & & & & & & & 2 & $4 \%$ \\
\hline More Scholarship Information & $0 \%$ & & 2 & 2 & $3 \%$ & & & & & & & & & & & & \\
\hline More Tutoring & $\mathbf{0 \%}$ & & 2 & & & & & 2 & $4 \%$ & & & & & & & & \\
\hline Other (6 miscellaneous comments) & $1 \%$ & & $\underline{6}$ & & & 2 & $4 \%$ & 2 & $4 \%$ & & & & & & & 2 & $4 \%$ \\
\hline & & $\underline{11 \%}$ & $\underline{46}$ & & & & & & & & & & & & & & \\
\hline total & & $\overline{100 \%}$ & $412 \overline{41}$ & \begin{tabular}{|l|l}
2 & 59 \\
\end{tabular} & & 54 & & 44 & & 55 & & 62 & & 67 & & 71 & \\
\hline Comments per Student & & & 1. & 0.8 & & 0.7 & & 0.9 & & 0.9 & & 1.0 & & 1.1 & & 1.4 & \\
\hline Total Number of Graduates & & & 42 & \begin{tabular}{l|l|}
7 & 71 \\
\end{tabular} & & 74 & & 49 & & 58 & & 61 & & 63 & & 51 & \\
\hline
\end{tabular}


For many years the Department of Accounting used two nationally normed testing measures in order to admit students into the major and to permit students to graduate as accounting majors. The APAT, which was used in the major admission process, is no longer available as a means of helping to assure the quality of the incoming majors. The ATAG was required for graduation. Prompted by the student exit interviews, the department reconsidered the use of the ATAG test as a graduation requirement. Although not convinced that the test was too stressful, the faculty abandoned its use because this test has not been updated or re-normed for many years. To replace these tests, which helped to support the quality of the accounting major, the standard of academic performance for admission into the program was raised. A student must now have a 2.70 or higher overall GPA and a 2.70 or higher GPA in 9 hours or more of upper-level accounting courses in order to be admitted into the accounting program, rather than the previous 2.50 GPA in accounting courses that could include Principles. The Accounting Department also is considering the possibility of another assessment instrument as a graduation requirement.

Prompted by the role that the internship program played in the placement process and feedback from the professional community regarding the importance of internships, an internship requirement has been added to the accounting major requirements. Students who are working in the accounting field have the opportunity for their employer to coordinate with the university internship office in order to qualify their work experience for the internship requirement.

Advising has recently been changed for the University as a whole. Rather than seeking advising as desired or when needed for admission to a particular class, each student must participate in a "mandatory" advising session each semester from first semester freshman year to the last semester prior to graduation. This cradle-to-grave approach has resulted in more consistent and valuable advising during the early years, but the necessity of this approach during the upper-level period, when students have matured and know what is required of them, is questionable. In order to implement this system almost all faculty members are heavily involved in advising. Accounting Department faculty would like to see this policy amended to recognize the different needs of lower-level and upper-level students and the different desires and skills of each faculty member.

Of the negative points addressing issues outside of the Department of Accounting, Accounting Department faculty do not necessarily agree with student assertions that management course requirements are excessive, but they remain sensitive to the need for continuous curriculum review. Regarding the Business Ethics course, in response to the student complaints the Accounting Department has revised the requirement so that the student may take a business ethics course in the Management Department rather than the Philosophy Department. This was possible because of the AACSB changes reducing the need for credits outside the business school. Lastly, there is agreement that technology and technological support is lacking but due to budgetary constraints beyond the purview of the Accounting Department, technology continues to be behind the curve, as does technological support.

\section{AUTHOR INFORMATION}

Barbara Stewart, professor of accounting, joined Towson University in the fall of 1988 and retired in the summer of 2008. Her interests focused on financial accounting, accounting education and accounting history. Dr. Stewart earned her bachelor's degree at Barnard College and Ph.D. at Columbia University. She served on the Maryland State Board of Public Accountancy and on the Board of Directors of the Maryland Association of Certified Public Accountants. Dr. Stewart is a recipient of the Elijah Watt Sells Silver Medal and of the MACPA Outstanding Accounting Educator Award.

Charles Martin, professor of accounting, joined Towson University in the fall of 1988. His research interests focus on personal financial planning, financial auditing, financial accounting, accounting education and finance. A certified public accountant in the State of Maryland, Dr. Martin is also a licensed realtor, investment adviser, and general securities investment representative. He holds his doctorate in accounting and finance and his master's in finance from The George Washington University. In 1999, Dr. Martin received the highest honor bestowed on an academic accounting professional by the Maryland Association of Certified Public Accountants when he was recognized as the "1999 Outstanding Accounting Educator" for the State of Maryland. 
Lamont Steedle, professor of accounting, joined Towson University in the fall of 1990 and retired in the summer of 2008. He earned his masters and doctorate from Penn State University. His research interests focused on managerial accounting and accounting education. He served as Department of Accounting chair for 14 years and led the department through its initial AACSB accreditation.

\section{REFERENCES}

1. Doll, P.A., K.W. Jacobs. 1988. The Exit Interview for Graduating Seniors. Teaching of Psychology 15 (4): $213-214$.

2. Herring, H.C. and C.D. Izard. 1992. Outcomes Assessment of Accounting Majors. Issues in Accounting Education Spring: $1-17$.

3. Louise, A., K.R. Mares, and E.V. Calkins. 1986. Exit Interviews Reveal Why Students Leave a BA-MD Degree Program Prematurely. College and University Fall: 35 - 47.

4. Nichols, J.O. 1991. A Practitioner's Handbook for Institutional Effectiveness and Student Outcomes Assessment Implementation. New York, NY: Agathon Press.

5. Stivers, B. P., J.E. Campbell, and H.M. Hermanson. 2000. An Assessment Program for Accounting: Design, Implementation, and Reflection. Issues in Accounting Education V.15 Issue 4: 553 - 581.

\section{NOTES}

